\title{
Can interfero-polarimetry constrain extended atmospheres' models?
}

\author{
K. Rousselet-Perraut \\ Observatoire de Haute-Provence, F-04870 Saint-Michel l'Observatoire, France \\ e-mail: Karine.Perraut@obs.ujf-grenoble.fr
}

Received April 24, 1997; accepted February 9, 1998

\begin{abstract}
Adding a polarimetric mode to a stellar interferometer enables to extend its exploration capacities for extended circumstellar environments in which intrinsic polarization becomes a non negligible observable. A general formalism is developed to map intensities and corresponding visibilities in polarized light for any extended atmosphere. To constrain matter distributions in scattering hot envelopes, an instrumental accuracy of $1 \%$ on the visibility is required, which is compatible with the performances of existing instruments. This implies an envelope flux contribution of $10-15 \%$ and limiting visual magnitudes $M_{v}$ of 4 in a monospeckle mode and of $8-10$ in multispeckle modes, which allows to contemplate studying several hot stars by this interfero-polarimetric technique. Finally, a specific instrumentation and an adapted methodology are mandatory: observations at small spatial frequencies $\left(B / \lambda \approx 1 /\left(10 R^{*}\right)\right.$ where $R^{*}$ is the stellar radius), simultaneous recordings of the polarized interferograms and an accurate calibration of the instrumental polarization. This technique can be applied to arrays of few telescopes (such as the Very Large Telescope Interferometer - VLTI) as well as to aperture synthesis arrays. Besides, such a polarimetric equipment must be advocated for the future instruments since it greatly reduces the instrumental bias.
\end{abstract}

Key words: techniques: interferometric; polarimetric stars: atmospheres; imaging

\section{Introduction}

Mass-loss usually occurs in stars and involves their outer layers (stellar winds) as well as their internal structures (energy responsible for mass flux). For hot stars (type O, A, B, WR) and especially for Be stars, despite various

Send offprint requests to: K. Rousselet-Perraut observational methods (IR excess, UV lines, ...), massloss is relatively misunderstood since matter distributions in the envelopes are not well known.

Constrains to mass-loss models can be obtained by measuring intrinsic polarization commonly attributed to Thomson scattering by the near totally ionized envelope (Serkowski 1968, 1970). However by classical polarimetric techniques which measure the flux averaged on the object surface, this leads to very constraining accuracies since the polarization degree is small. Besides these usual techniques detect polarization effects inside morphologically dissymmetric envelopes only. On the contrary, an interferometer equipped with a polarimetric mode (called an interfero-polarimeter) allows to study polarization inside any envelope, whatever its symmetry degrees. In practice, the measurement of the fringe visibility through a linear polarizer parallel then perpendicular to the interferometric baseline enables to measure the distortion of the scattering atmosphere seen through the linear polarizer.

Such interfero-polarimetric observations have already been done (Hanbury Brown et al. 1974; Vakili 1981; Rousselet-Perraut et al. 1997a) and have shown the great interest in developing adapted astrophysical models. Such models are mandatory to interpret the recorded data and, above all, to efficiently carry out future observations (choice of objects and adapted interferometer configurations for instance). Now, only one model has been developed for Wolf-Rayet stars (Cassinelli \& Hoffmann 1975) and no general formalism exists to describe the interfero-polarimetric observables. Within the context of the definition of the VLTI focal instrumentation (Paresce et al. 1996), we try to design the instrumental requirements as well as the observational constrains of interferopolarimetry. Thus we develop a general analytical method providing the intensity maps and the corresponding visibilities recorded in polarized light for any scattering environment. In this model, the geometry and the flux contribution of the envelope, the polarizer orientation and the source function can be parametrized. Since we first aim at 
defining the interferometric observables, we only consider pure single Thomson scattering (Sect. 2). In Sect. 3, we apply our model to a spherical star surrounded by a spherical envelope and we discuss the effect on the visibility of the envelope diameter, its flux contribution and its electron distribution. In Sect. 4, we consider a spherical star surrounded by an ellipsoidal envelope and we study the visibility dependence on flattening and electron distribution. Finally, we discuss the hypotheses of our modeling and the instrumental and observational requirements of interfero-polarimetry, which leads us to propose a specific instrumentation. To conclude we give some astrophysical applications which would be very attractive with future optical aperture synthesis arrays.

\section{Synthetic intensity maps and visibilities in polarized light}

\subsection{General formalism and basic equations}

Thomson scattering occurs when an electromagnetic field impinges upon a free electron. The latter is accelerated and reradiates the incident radiation. This scattered radiation can be observed outside the propagation axis of the incident field. Whatever the polarization state of the incident field, the polarization degree of the scattered radiation varies with its direction (Collett 1993). In the following we assume that the natural (or unpolarized) starlight is scattered by free electrons of the hot envelope only and we do not consider multiple scattering (hypothesis of pure single Thomson scattering).

Figure 1 shows the reference system of Cartesian coordinates $(x, y, z)$ centered on the star $O .(O z)$ is the rotation axis and the plane $(O x z)$ includes the line of sight. A point $M$ of the envelope is characterized by three spherical coordinates $(r, \theta, \varphi)$ in this system and by $\left(r, \theta^{\prime}, \varphi^{\prime}\right)$ in the system $\left(x^{\prime}, y^{\prime}, z^{\prime}\right)$ related to the sight direction $\left(O x^{\prime}\right)$ and where $\left(O y^{\prime}\right)$ coincides with $(O y)$.

The angle between $O M$ and the line of sight $\left(O x^{\prime}\right)$ is the scattering angle $\chi$. From Fig. 1 and by denoting $i$ the axial inclination (i.e. the angle $x O z^{\prime}$ ) we obtain:

$\cos \chi=\sin \theta \cos \varphi \sin i-\cos \theta \cos i=\sin \theta^{\prime} \cos \varphi^{\prime}$.

From the expressions of the transverse components of the incident field and from the motion equations of the free electron, we find the scattered intensity at $M$ (Collett 1993; Brown \& McLean 1977):

$I(r, \theta, \varphi)=\frac{3 \sigma_{\mathrm{T}}}{16 \pi} n(r, \theta) \frac{1+\cos ^{2} \chi}{r^{2}} I_{0}$

where $I_{0}$ denotes the isotropic light emitted by $O$ and $n(r, \theta)$ the electron distribution at $M . \sigma_{\mathrm{T}}$ is the independent wavelength Thomson cross section (Collett 1993).

Characteristically the scattered intensity is maximal along the axis of the incident radiation (on-axis scattering) and is minimal perpendicularly to this direction (off-axis scattering).

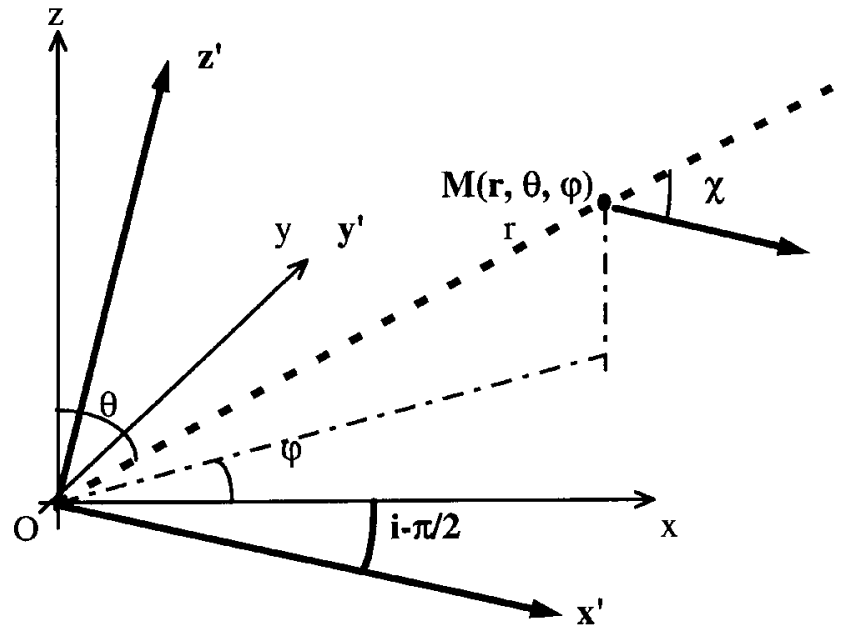

Fig. 1. Each point $M$ of the scattering envelope is characterized by three spherical coordinates $(r, \theta, \varphi)$ in the Cartesian system $(x, y, z)$ centered on the star $O .(O z)$ is the axis of rotation and the plane $(O x z)$ includes the line of sight. $M$ is also defined by $\left(r, \theta^{\prime}, \varphi^{\prime}\right)$ in the system $\left(x^{\prime}, y^{\prime}, z^{\prime}\right)$ related to the sight direction $\left(O x^{\prime}\right) .\left(O y^{\prime}\right)$ is coincident with $(O y)$ and $i$ denotes the axial inclination. The scattering angle at $M$ is $\chi$

By convention, the local degree of polarization at $M$ is defined by the difference between the maximal and minimal intensities normalized to the sum of these intensities. Hence:

$p(\theta, \varphi)=\frac{1-\cos ^{2} \chi}{1+\cos ^{2} \chi}$

The degree of polarization does not depend on the radial position $r$. It equals 0 for on-axis scattering and 1 for off-axis scattering. The scattered radiation is partially polarized for intermediate angles.

The direction of polarization is perpendicular to the scattering plane defined by $O M$ and $\left(O x^{\prime}\right)$. Thus it lies along $O M \times O x^{\prime}$ (where $\times$ denotes the vectorial product). In the plane $\left(y^{\prime} \mathrm{O} z^{\prime}\right)$ the polarization is normal to $O M$ and therefore tangential to the envelope.

\subsection{Intensity maps in polarized light}

We aim at obtaining the intensity maps in the plane $\left(y^{\prime} \mathrm{O} z^{\prime}\right)$ for various configurations of the interferopolarimeter (with and without linear polarizer). For convenience we assume that the two sets of coordinates are identical ( $i=90^{\circ}$ in Fig. 1) (See discussion in Sect. 5.1). For each point $M_{0}\left(0, r_{0}, \sin \theta_{0}^{\prime}, r_{0} \cos \theta_{0}^{\prime}\right)$ of $\left(y^{\prime} \mathrm{O} z^{\prime}\right)$ we compute the intensity and the degree of polarization of the scattered radiation integrated on the sight column. This column lies along $\left(O x^{\prime}\right)$ and is limited by the envelope dimension $R_{\text {env }}$ along this direction. The integrated intensity at $M_{0}$ is given by:

$\bar{I}\left(r_{0}, \theta_{0}\right)=\int_{r_{0}}^{R_{\mathrm{env}}} I(r, \theta(r), \varphi(r)) \mathrm{d} r$ 
with $\theta(r)=\operatorname{Arccos}\left(\frac{r_{0} \cos \theta_{0}}{r}\right)$ and $\varphi(r)=$ $\operatorname{Arcsin}\left(\frac{r_{0} \sin \theta_{0}}{r \sin \theta(r))}\right)$. The integrated degree of polarization is given by:

$\bar{p}\left(r_{0}, \theta_{0}\right)=\int_{r_{0}}^{R_{\mathrm{env}}} p(\theta(r), \varphi(r)) \mathrm{d} r$

By symmetry the direction of polarization at $M_{0}$ is tangential to the envelope and thus characterized by the angle $\theta_{0}^{\prime}-\pi / 2$ with respect to $\left(O z^{\prime}\right)$.

We describe the polarization state at $M_{0}$ (which is partially polarized along the angular direction $\theta_{0}^{\prime}-\pi / 2$ ) by a four-element Stokes vector (Collett 1993):

$$
\begin{aligned}
\mathbf{S}\left(M_{0}\right)=\left[\left(1-\bar{p}\left(M_{0}\right)\right) \cdot \mathbf{S}_{\text {nat }}\right. & \\
& \left.+\bar{p}\left(M_{0}\right) \cdot \mathbf{S}_{\mathrm{rect}\left(\theta_{0}^{\prime}-\pi / 2\right)}\left(M_{0}\right)\right] \bar{I}\left(M_{0}\right)
\end{aligned}
$$

where $\mathbf{S}_{\text {nat }}=[1 ; 0 ; 0 ; 0]$ is the Stokes vector of natural light and $\mathbf{S}_{\text {rect }\left(\theta_{0}^{\prime}-\pi / 2\right)}\left(M_{0}\right)=\left[1 ;-\cos \left(2 \theta_{0}^{\prime}\right) ;-\sin \left(2 \theta_{0}^{\prime}\right) ; 0\right]$ the Stokes vector of totally linearly polarized light along the angular direction $\theta_{0}^{\prime}-\pi / 2$.

The first component of $\mathbf{S}\left(M_{0}\right)$ is the total intensity of the radiation. It equals $\bar{I}\left(M_{0}\right)$ in natural light (Eq. 6). If the scattering envelope is now observed through a linear polarizer, the Stokes vector after the polarizer $\mathbf{S}^{\prime}\left(M_{0}\right)$ is found from Eq. (6) by a linear transformation, as follows:

$\mathbf{S}^{\prime}\left(M_{0}\right)=[M] . \mathbf{S}^{\prime}\left(M_{0}\right)$

with $[M]$ the Mueller matrix of the linear polarizer (Collett 1993):

$[M]=\frac{P_{t}^{2}}{2}\left[\begin{array}{cccc}1 & \cos (2 \alpha) & \sin (2 \alpha) & 0 \\ \cos (2 \alpha) & \cos ^{2}(2 \alpha) & \cos (2 \alpha) \sin (2 \alpha) & 0 \\ \sin (2 \alpha) & \cos (2 \alpha) \sin (2 \alpha) & \sin ^{2}(2 \alpha) & 0 \\ 0 & 0 & 0 & 0\end{array}\right]$ (8)

where $P_{t}$ denotes the transmission of the linear polarizer and $\alpha$ its azimuth.

For $\alpha=0$ and $\alpha=\pi / 2$ we obtain two polarized intensity maps. They correspond to a linear polarizer parallel then perpendicular to $\left(O z^{\prime}\right)$ respectively. They are denoted by the subscripts // and $\perp$ respectively. The intensity maps are given by the first components of the resulting Stokes vectors (Eq. 7):

$$
\begin{aligned}
& \bar{I}_{/ /}\left(M_{0}\right)=\frac{P_{t}^{2}}{2}\left[1-\bar{p}\left(M_{0}\right) \cos \left(2 \theta_{0}^{\prime}\right)\right] \bar{I}\left(M_{0}\right) \\
& \bar{I}_{\perp}\left(M_{0}\right)=\frac{P_{t}^{2}}{2}\left[1+\bar{p}\left(M_{0}\right) \cos \left(2 \theta_{0}^{\prime}\right)\right] \bar{I}\left(M_{0}\right) .
\end{aligned}
$$

\subsection{Visibilities and angular diameters in polarized light}

A stellar interferometer yields a measurement of the amplitude and the phase of the object spatial coherence function at the spatial frequency $B / \lambda$ (where $B$ is the interferometric baseline and $\lambda$ the wavelength). Furthermore, we call "visibility" the modulus of the Fourier Transform of this intensity distribution at the spatial frequency $B / \lambda$ normalized to the value at the zero frequency (Françon 1966):

$V(B / \lambda)=\left|\frac{\tilde{\bar{I}}(B / \lambda)}{\tilde{\bar{I}}(0)}\right|$

where $V$ denotes the visibility and ${ }^{\sim}$ the Fourier transform.

For convenience we assume that the baseline lies along $\left(O z^{\prime}\right)$. Each intensity map provides a polarized visibility $\left(V_{\text {nat }}, V_{/ /}, V_{\perp}\right)$. Assuming the baseline along $\left(O y^{\prime}\right)$ leads to three other visibilities $\left(V_{\text {nat }}^{\prime}, V_{/ /}^{\prime}, V_{\perp}^{\prime}\right)$. For spherical envelopes these visibilities satisfy $V_{\text {nat }}^{\prime}=V_{\text {nat }}, V_{/ /}^{\prime}=V_{\perp}$ and $V_{\perp}^{\prime}=V_{/ /}$and changing the baseline is useless. But for non axi-symmetric envelopes, each baseline provides independent visibilities which enable to constrain the envelope geometry (See Sects. 4.3 and 5.5).

Considering the star and the envelope as uniform disks we can fit each visibility curve versus spatial frequency with the function:

$$
V(B / \lambda)=\left|F_{*} \frac{2 J_{1}\left(\pi \phi^{*} B / \lambda\right)}{\pi \phi^{*} B / \lambda}+F_{\mathrm{env}} \frac{2 J_{1}\left(\pi \phi_{\mathrm{env}} B / \lambda\right)}{\pi \phi_{\mathrm{env}} B / \lambda}\right|
$$

where $J_{1}$ is the Bessel function of first degree, $\phi^{*}$ the angular diameter of the star, $F_{*}$ the flux contribution of the star, $F_{\text {env }}$ the flux contribution of the envelope and $\phi_{\text {env }}$ the angular diameter of the envelope.

Each polarized visibility thus provides an equivalent angular diameter $\left(\Phi_{\text {nat }}, \Phi_{/ /}, \Phi_{\perp}\right) . \Phi_{\perp}$ is larger than $\Phi_{/ /}$ and $\Phi_{\text {nat }}$ is between (Eq. 9).

To study the effect of polarization on visibility we define the degree of polarized visibility $P_{V}$ by:

$P_{V}=\frac{V_{/ /}-V_{\perp}}{V_{\text {nat }}}$.

\section{Case of spherical envelopes}

We aim at determining three parameters of the envelope: its relative flux $F_{\text {env }}$, its angular diameter $\phi_{\text {env }}$ (Eq. 11) and its radial distribution of electron expressed by $n(r)=$ $n_{0}\left(\frac{r}{R^{*}}\right)^{k} . R^{*}$ denotes the stellar radius, $n_{0}$ the electron density at the stellar surface and $k$ the exponent of the distribution. For this purpose, we study various interferopolarimetric observables.

\subsection{Polarized intensity maps and visibilities}

For a spherical envelope, the three intensity maps are different (Figs. 2-4). Each polarized map is flattened along the polarizer direction. Its shape is identical whatever the polarizer direction and only its orientation varies. That's why classical polarimetry fails to detect any difference in such cases. The Fourier transforms of Figs. 2-4 provide polarized visibilities whose differences can be large $(\approx 10 \%)$ for small spatial frequencies (i.e. for $B / \lambda$ equal to $1 /(10$ $\left.R^{*}\right)$ ). Such spatial frequencies correspond to large structures of the observed object and to short baselines from 


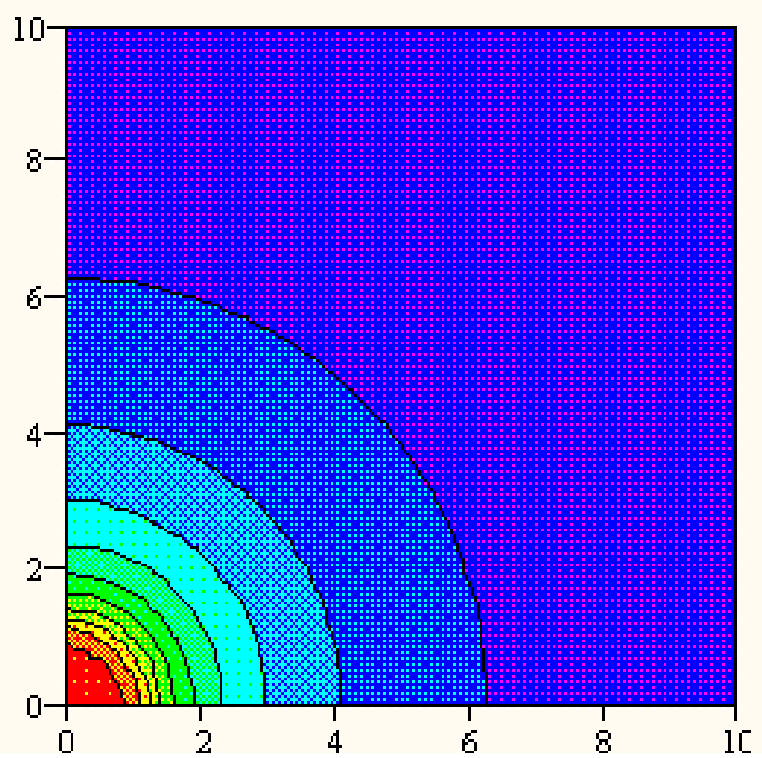

Fig. 2. Intensity map in natural light for an uniform scattering envelope of 10 stellar radius. The gap between two iso-contours equals 65 Intensity Units

the ground $(10-20$ meters at $0.6 \mu \mathrm{m}$ for a stellar radius of $0.5-1$ mas): the object is partially resolved. These differences between the polarized visibilities are obviously characteristic of the exponent $k$. But, in practice, these differences are greatly attenuated by the dominant flux contribution of the star $\left(F_{*}\right)$ and by its large visibility (since $\phi^{*}$ is often less than 1 mas, $\frac{2 J_{1}\left(\pi \phi^{*} B / \lambda\right)}{\pi \phi^{*} B / \lambda}$ is close to $100 \%)$. Thus the polarized visibilities are not sufficient to strongly constrain our parameters $\left(F_{\text {env }}, \phi_{\text {env }}, k\right)$ and a differential study is mandatory (See Sect. 3.3).

\subsection{Equivalent uniform disks}

For various configurations of $\left(F_{\mathrm{env}}, \phi_{\mathrm{env}}, k\right)$ we fit the visibility curves versus spatial frequency from Eq. (11) (hypothesis of uniform disk). We compute the ratio of the polarized angular diameters $\Phi_{\perp} / \Phi_{/ /}$and the relative variation of angular diameter with polarization $\delta \Phi=$ $\left(\Phi_{\perp}-\Phi_{/ /}\right) / \Phi_{\text {nat }}$. We check that the larger the exponent $k$, the smaller the angular diameter (Table 1). For $F_{\text {env }}=30 \%$ when $k$ varies from 0 to $3, \Phi_{\perp} / \Phi_{/ /}$increases by 0.051 and $\delta \Phi$ by $4.8 \%$. Considering a typical error of 0.1 mas on the angular diameters, we obtain an error of $0.15-0.20$ on $\Phi_{\perp} / \Phi_{/ /}$and of $15-20 \%$ on $\delta \Phi$. Therefore polarized angular diameters do not allow to differentiate $k$ even for a high envelope flux of $30 \%$. For more realistic smaller flux (See Sect. 5.3), the variations of $\Phi_{\perp} / \Phi_{/ /}$ and $\delta \Phi$ with $k$ are clearly reduced and we conclude that $F_{\text {env }}$ and $\phi_{\text {env }}$ can be determined from the natural visibility curve. $F_{\text {env }}$ is obtained at large spatial frequencies (i.e. few tenths meters) where the envelope contribution is negligible. The resulting visibility is $F^{*} \cdot \frac{2 J\left(\pi \phi^{*} B / \lambda\right)}{\pi \phi^{*} B / \lambda}$ (Eq. 11)

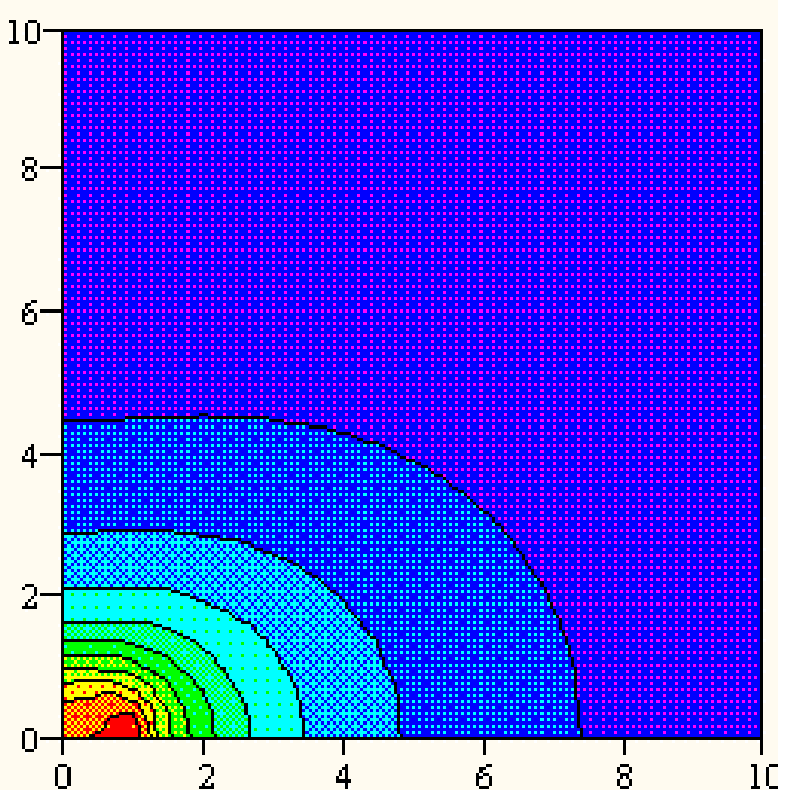

Fig. 3. Intensity map in polarized light (with a linear polarizer perpendicular to the baseline) for an uniform scattering envelope of 10 stellar radius. The gap between two iso-contours equals 72 Intensity Units

Table 1. Polarized angular diameters, ratios of polarized angular diameters and relative variation of angular diameters with polarization for a spherical envelope of 10 stellar radii responsible for $30 \%$ of the total flux and for various exponents $k$ of the electron distribution

\begin{tabular}{lcccccc}
$k$ & $F_{\text {env }}$ & $\Phi_{\text {nat }}$ & $\Phi_{/ /}$ & $\Phi_{\perp}$ & $\Phi_{\perp} / \Phi_{/ /}$ & $\delta \Phi=\frac{\Phi_{/ /}-\Phi_{\perp}}{\Phi_{\text {nat }}}$ \\
\hline 0 & 30 & 1.51 & 1.48 & 1.53 & 1.034 & $3.3 \%$ \\
0.5 & 30 & 1.47 & 1.44 & 1.50 & 1.042 & $4.1 \%$ \\
1 & 30 & 1.43 & 1.39 & 1.46 & 1.050 & $4.9 \%$ \\
2 & 30 & 1.32 & 1.27 & 1.36 & 1.071 & $6.8 \%$ \\
3 & 30 & 1.24 & 1.18 & 1.28 & 1.085 & $8.1 \%$ \\
\hline
\end{tabular}

with $\frac{2 J\left(\pi \phi^{*} B / \lambda\right)}{\pi \phi^{*} B / \lambda} \approx 1 . \phi_{\text {env }}$ can be determined by considering the envelope as a uniform disk $(k=0)$. However this method can lead to an over-estimation of $\phi_{\text {env }}$ if $k$ exceeds 2 (over-estimation of about $13 \%$ for $k=3$ and $\left.F_{\text {env }}=30 \%\right)$.

\subsection{Degree of polarized visibility $P_{V}$}

The variations of $P_{V}$ versus spatial frequency have the same shapes whatever $F_{\text {env }}$. They are also very small whatever the envelope radius $R_{\text {env }}$. On the contrary, these curves are obviously characteristic of the exponent $k$. Provided that $F_{\text {env }}$ is large enough (i.e. $\geq 15 \%$ ), we can disentangle all the exponents $(0,0.5,1,2,3)$ if the accuracy on the visibility equals $0.1 \%$ (Fig. 5 ). An accuracy of 


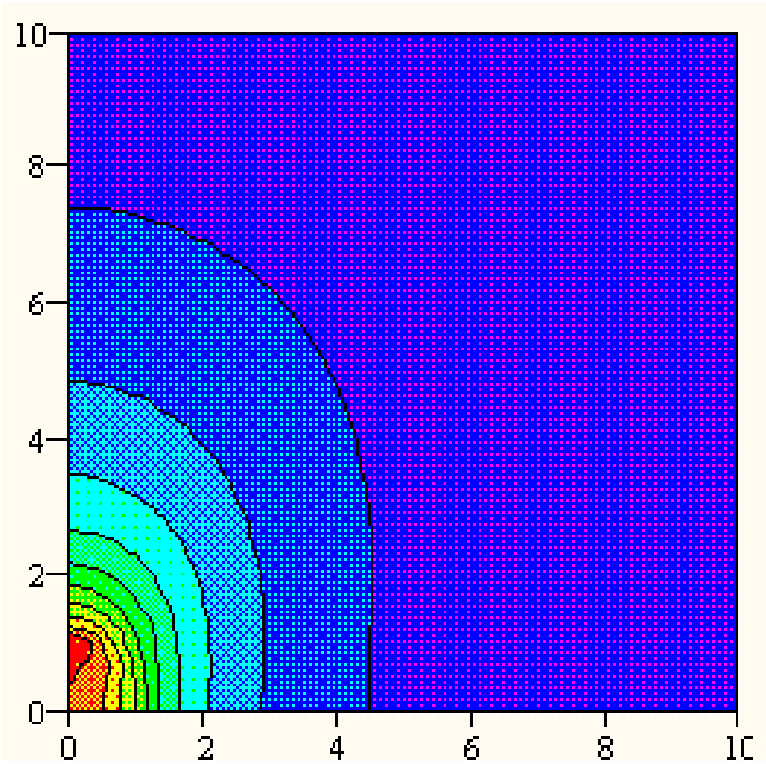

Fig. 4. Intensity map in polarized light (with a linear polarizer parallel to the baseline) for an uniform scattering envelope of 10 stellar radius. The gap between two iso-contours equals 72 Intensity Unit

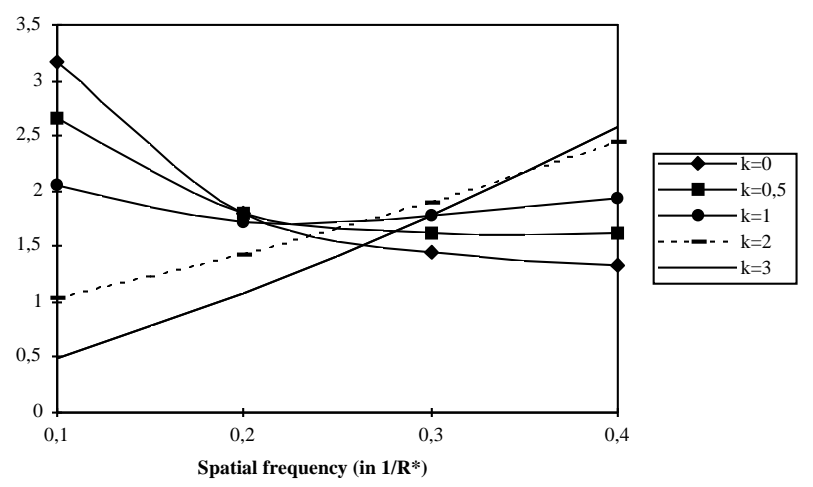

Fig. 5. Degree of polarized visibility versus spatial frequency for an envelope of 10 stellar radii responsible for $15 \%$ of the total flux and for various exponent $k$ of the electron distribution

$1 \%$ on the visibility is enough to put a lower limit to $k$. The accuracy on $k$ is about 0.5 for $2<k \leq 3$ and about 0.25 for $1 \leq k \leq 2$.

\section{Case of ellipsoidal envelopes}

The major phenomenon producing intrinsic polarization in Be and shell stars is Thomson scattering of the starlight by an oblate rotationally symmetric envelope. Such envelopes are usually studied by photo- and spectropolarimetry (Wood et al. 1993; Wood et al. 1996): the integrated degree of polarization is of the order of $1 \%$ and rarely exceeds $1.5 \%$ (Coyne 1976). We have modeled the observations of such objects (star + a flattened envelope) by interfero- polarimetry. We have considered electron

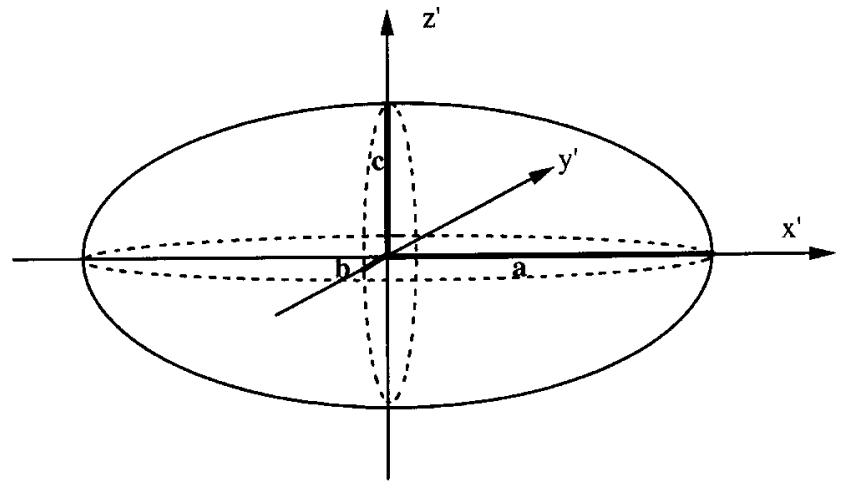

Fig. 6. Definition of the dimensional parameters of an ellipsoidal envelope. The system $\left(x^{\prime}, y^{\prime}, z^{\prime}\right)$ related to the sight direction $\left(O x^{\prime}\right)$ is assumed to be coincident with the stellar system $(x$, $y, z)$ defined in Fig. 1

distributions expressed by $n_{0} /\left(x^{\prime 2} / a^{2}+y^{\prime 2} / b^{2}+z^{\prime 2} / c^{2}\right)^{k}$ where $a, b$ and $c$ are the envelope dimensional parameters. $a$ lies along $\left(O x^{\prime}\right), b$ along $\left(O y^{\prime}\right)$ and $c$ along $\left(O z^{\prime}\right)$ (Fig. 6). For axi-symmetric envelopes, $a$ equals $b . k$ is the exponent of the distribution law and $\varepsilon=b / c$ is the envelope flattening. We aim at constraining $F_{\text {env }}, \varepsilon$ and $k$ by interfero-polarimetry.

\subsection{Polarized visibilities}

If we consider the polarized visibilities normalized to the natural one, their variations versus spatial frequency are too small (i.e. $<0.3 \%$ ) for determining $k$ or $\varepsilon$. Moreover at small spatial frequencies $\left(\approx 1 /\left(10 R^{*}\right)\right)$, the comparison between the normalized polarized visibilities do not enable to differentiate $k$ except for $F_{\text {env }}=30 \%$ (See discussion in Sect. 5.3) and for an accuracy of $0.1 \%$ on the visibility.

\subsection{Equivalent uniform disks}

The variations of $\Phi_{\perp} / \Phi_{/ /}$and $\delta \Phi$ clearly depend upon $k$ (Tables 2 and 3 ) and $\varepsilon$ (Table 4). They are larger for an extended envelope (Tables 2 and 3 ) and for a large $F_{\text {env }}$. Even for $F_{\mathrm{env}}=30 \%$, these variations are too small for determining $k$ or $\varepsilon$ if we consider a typical error of 0.1 mas on angular diameters. In fact when $k$ varies from 0 to $3, \Phi_{\perp} / \Phi_{/ /}$increases by 0.087 and $\delta \Phi$ by $8.1 \%$ (Table 2 ). When $\varepsilon$ varies from 0.5 to 4 , the variations of $\Phi_{\perp} / \Phi_{/ /}$ equals 0.029 and those of $\delta \Phi$ equals $2.81 \%$ (Table 4 ). The values have to be compared with error bars given in Sect. 2.1. $(0.15-0.2$ and $15-20 \%$ respectively). As shown for spherical envelopes, angular dimensions and thus $\varepsilon$ can be determined from the natural visibility.

\subsection{Degree of polarized visibility $P_{V}$}

The variations of $P_{V}$ versus spatial frequency have the same shapes for a variation of $k$ as for a variation of $\varepsilon$ 
Table 2. Polarized angular diameters, ratios of polarized angular diameters and relative variation of angular diameters with polarization for an ellipsoidal envelope whose dimensional parameters are respectively 10,10 and 20 stellar radii $(\varepsilon=0.5)$ and responsible for $30 \%$ of the total flux and for various exponents $k$ of the electron distribution

\begin{tabular}{lllllll}
$k$ & $F_{\text {env }}$ & $\Phi_{\text {nat }}$ & $\Phi_{/ /}$ & $\Phi_{\perp}$ & $\Phi_{\perp} / \Phi_{/ /}$ & $\delta \Phi=\frac{\Phi_{/ /}-\Phi_{\perp}}{\Phi_{\text {nat }}}$ \\
\hline 0 & 30 & 2.34 & 2.28 & 2.40 & 1.053 & $5.1 \%$ \\
0.5 & 30 & 2.19 & 2.12 & 2.26 & 1.066 & $6.4 \%$ \\
1 & 30 & 1.98 & 1.91 & 2.07 & 1.084 & $8.1 \%$ \\
2 & 30 & 1.53 & 1.44 & 1.63 & 1.132 & 12.4 \\
3 & 30 & 1.21 & 1.14 & 1.30 & 1.140 & $13.2 \%$ \\
\hline
\end{tabular}

Table 3. Polarized angular diameters, ratios of polarized angular diameters and relative variation of angular diameters with polarization for an ellipsoidal envelope whose dimensional parameters are respectively 10,10 and 7.5 stellar radii $(\varepsilon=1.33)$ and responsible for $30 \%$ of the total flux and for various exponents $k$ of the electron distribution

\begin{tabular}{llllllc}
$k$ & $F_{\text {env }}$ & $\Phi_{\text {nat }}$ & $\Phi_{/ /}$ & $\Phi_{\perp}$ & $\Phi_{\perp} / \Phi_{/ /} \delta \Phi=\frac{\Phi_{/ /}-\Phi_{\perp}}{\Phi_{\text {nat }}}$ \\
\hline 0 & 30 & 1.50 & 1.47 & 1.53 & 1.041 & $4.0 \%$ \\
0.5 & 30 & 1.47 & 1.44 & 1.50 & 1.042 & $4.1 \%$ \\
1 & 30 & 1.43 & 1.39 & 1.46 & 1.050 & $4.9 \%$ \\
2 & 30 & 1.33 & 1.28 & 1.36 & 1.063 & $6.0 \%$ \\
3 & 30 & 1.24 & 1.19 & 1.27 & 1.067 & $6.5 \%$ \\
\hline
\end{tabular}

(Fig. 7). Given a flattening $\varepsilon$, we can differentiate all the exponents $k$ provided that the spatial frequency is small enough $\left(\approx 1 /\left(10 R^{*}\right)\right), F_{\text {env }}$ attains $10 \%$ and the accuracy on the visibility equals $0.1 \%$. An accuracy of $1 \%$ on the visibility is enough to put a lower limit to $k$. The accuracy on $k$ clearly depends on the envelope geometry but it is generally better than 0.5 . Given an exponent $k$, using $P_{V}$ to determine $\varepsilon$ leads to a poor accuracy $(\approx 0.25$ for $F_{\text {env }}=10 \%$ ). To constrain the envelope geometry it is clearly better to use the high angular resolution and the capacity of Earth-rotation synthesis of the interferometer (See Sect. 5.5).

\section{Discussion}

\subsection{Hypotheses of our formalism}

Since we wanted to know if interfero-polarimetry is sensitive enough to detect polarization effects in an hot envelope, we have considered the most favorable case (maximal polarization rate) and evaluated the required accuracies (See Sect. 5.2). Thus we have limited our study to a
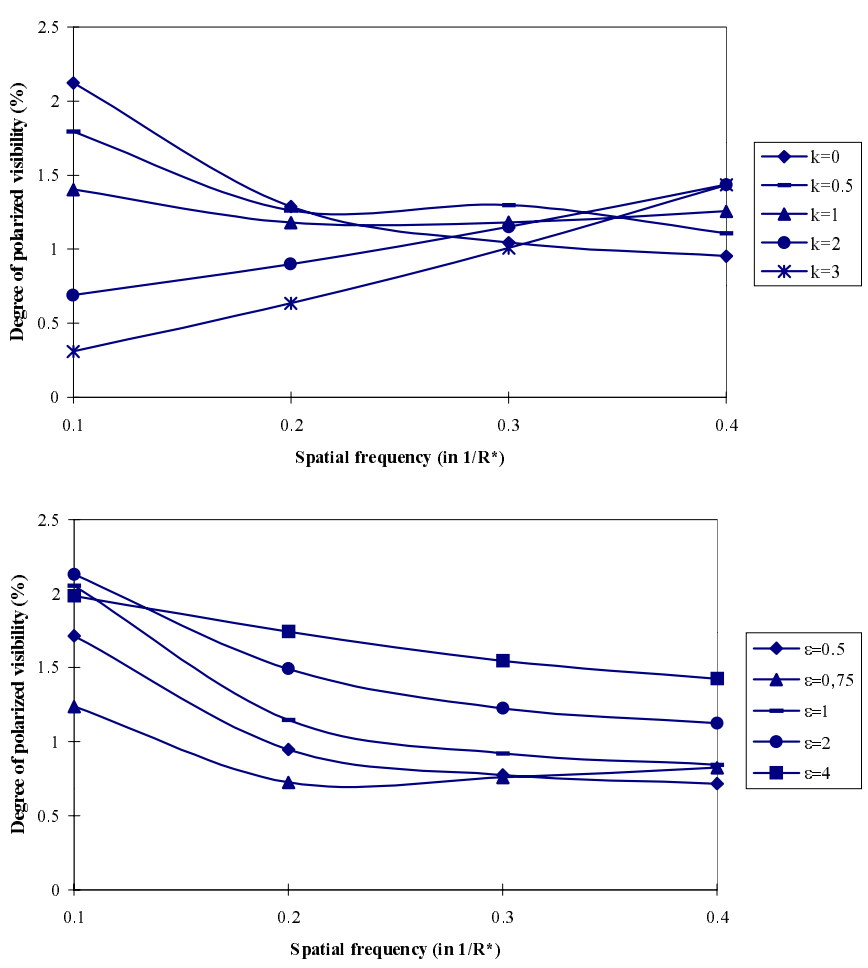

Fig. 7. Degree of polarized visibility versus spatial frequency for an ellipsoidal envelope responsible for $10 \%$ of the total flux: top) for various exponents $k$ of the electron distribution and for dimensional parameters of 10,10 and 7.5 stellar radii respectively $(\varepsilon=1.33)$, bottom) for various flattenings $\varepsilon$ and for an uniform envelope $(k=0)$

$90^{\circ}$ axial inclination and pure single Thomson scattering by considering optically thin approximations. In practice, because of high temperatures, hot envelopes are generally near totally ionized and absorption effects are thus not important. But near total ionization also implies multiple scattering which affects the polarization rate (Brown \& McLean 1977 and Wood et al. 1996). So, since our formalism is general, it must be applied in a near future to any axial inclination and to more realistic source functions (including opacities).

\subsection{Required accuracies}

It appears difficult to use polarized angular diameters to determine the electron distribution inside a circumstellar envelope whatever its shape. In fact it requires an accuracy on angular diameters of few $\mu$ as for a spherical envelope and of few tenths $\mu$ as for an ellipsoidal one whereas the best accuracy of the MARK III Interferometer equals about 0.05 mas (Mozurkewich et al. 1991) and that of the GI2T Interferometer equals about $0.1-0.15$ mas (Mourard et al. 1997). But, using the degree of polarized visibility is less constraining since whatever the shape of the envelope, an accuracy on visibility of $1 \%$ is enough to 
Table 4. Polarized angular diameters, ratios of polarized angular diameters and relative variation of angular diameters with polarization for an uniform ellipsoidal envelope $(k=0)$ responsible for $30 \%$ of the total flux for various flattenings $\varepsilon$

\begin{tabular}{ccccccccccc}
\hline$k$ & $a$ & $b$ & $c$ & $\varepsilon$ & $F_{\text {env }}$ & $\Phi_{\text {nat }}$ & $\Phi_{/ /}$ & $\Phi_{\perp}$ & $\Phi_{\perp} / \Phi_{/ /}$ & $\delta \Phi=\frac{\Phi_{/ /}-\Phi_{\perp}}{\Phi_{\text {nat }}}$ \\
\hline 0 & 10 & 10 & 2.5 & 4 & 30 & 1.47 & 1.42 & 1.51 & 1.063 & $6.12 \%$ \\
0 & 10 & 10 & 5 & 2 & 30 & 1.49 & 1.46 & 1.52 & 1.041 & $4.03 \%$ \\
0 & 10 & 10 & 7.5 & 1.33 & 30 & 1.50 & 1.47 & 1.53 & 1.041 & $4.00 \%$ \\
0 & 10 & 10 & 10 & 1 & 30 & 1.51 & 1.48 & 1.53 & 1.034 & $3.31 \%$ \\
0 & 10 & 10 & 15 & 0.75 & 30 & 1.92 & 1.88 & 1.97 & 1.048 & $4.69 \%$ \\
0 & 10 & 10 & 20 & 0.5 & 30 & 2.34 & 2.28 & 2.40 & 1.053 & $5.13 \%$ \\
\hline
\end{tabular}

put a lower limit to the exponent $k$ and an accuracy of $0.1 \%$ allows to distinguish the exponents $0,0.5,1,2,3$ if the envelope flux attains $15 \%$. Note that an accuracy of $1 \%$ is expected with the GI2T/REGAIN (Mourard et al. 1994 ) and an accuracy of $0.2 \%$ is obtained on the IOTA interferometer equipped with the FLUOR beam-combiner (Perrin 1996) thanks to a real-time photometric calibration explained by Roddier \& Roddier (1976).

\subsection{Astrophysical limitations}

Our study shows that interfero-polarimetry enables to determine matter distribution inside circumstellar envelopes provided that their flux contributions attain $10-15 \%$. Such contributions are characteristic of Be stars in the visible spectrum: it has been predicted by Poeckert \& Marlborough (1978) and observed by Stee et al. (1995) in interferometry. For B6-B9 stars and even for A0 stars, such contributions are realistic whereas for O stars larger contributions in the visible are expected. Interferopolarimetry can therefore be foreseen for these hot objects. Our technique cannot be extended to weaker flux since it implies a too severe accuracy on the visibility for the existing or planned instruments.

A signal-to-noise ratio (SNR) of 150 is required to reach an accuracy of $1 \%$ and a SNR of 1500 is needed to reach an accuracy of $0.1 \%$. For high visibilities $(\approx 0.85)$, the SNR in the photon limited case is given by (Percheron 1991 - See also Roddier \& Léna 1984 for the general expression):

$S N R=\frac{V}{2} \sqrt{N_{\mathrm{i}}} \sqrt{N_{\mathrm{s}}} \sqrt{\frac{\left\langle N_{\mathrm{ph}}\right\rangle}{2}}$

where $V$ is the recorded visibility, $N_{\mathrm{i}}$ the number of recorded images, $N_{\mathrm{s}}$ the number of speckles and $\left\langle N_{\mathrm{ph}}\right\rangle$ the average number of photo-events detected per elementary exposure and per speckle.

Now $\left\langle N_{\mathrm{ph}}\right\rangle$ is related to the visual magnitude $M_{v}$ by (Roddier \& Léna 1984):

$\left\langle N_{\mathrm{ph}}\right\rangle=T_{\text {interf }} \tau \sigma \Delta \lambda 10^{8-0.4 M_{V}}$ with $T_{\text {interf }}$ the instrumental throughput (detector and polarizer included), $\tau$ the exposure time in seconds, $\sigma$ the coherence area in $\mathrm{m}^{2}$ and $\Delta \lambda$ the optical bandwidth in $\mathrm{nm}$.

For the GI2T/REGAIN we shall assume that the visibility losses due to instrumental polarization attain $5 \%$ (Rousselet-Perraut 1996a), $\sigma=510^{-3} \mathrm{~m}^{2}, \tau=20 \mathrm{~ms}$ and $T_{\text {interf }}=0.5 \%$. We take $\Delta \lambda=5 \mathrm{~nm}$ and an integration time of $15 \mathrm{~min}$, which leads to $N_{\mathrm{i}}=45000$. The limiting visual magnitudes in mono- and multi-speckle modes are given in Table 5 . For adaptive optics modes, $N_{\mathrm{s}}=1$ and $\sigma$ is replaced by $s \pi R^{2}$ (Eq. 14) where s is the Strehl ratio and $R$ the telescope radius (Rousset et al. 1992). For the GI2T/REGAIN $s=0.1$ and $R=0.76 \mathrm{~m}$. For the VLTI, a Strehl ratio of 0.2 is expected in the visible and $R=0.9 \mathrm{~m}$. All these values show that a significant number of hot stars can be studied by the interfero-polarimetric technique.

\subsection{Instrumental device}

Our first observing run done with the GI2T Interferometer (Rousselet-Perraut et al. 1997a) on the Be star $\gamma$ Cassiopeiae has shown that: i) the instrumental polarization calibration is crucial and ii) the natural and polarized visibilities have to be simultaneously recorded to calibrate the temporal variation of the instrumental transfer function. The first point implies an accurate modeling and optimization of the instrumental design, especially in terms of coatings (Rousselet-Perraut et al. 1996a) as well as the calibration of the residual instrumental polarization. The second point requires the introduction of a polarimeter inside the classical focal instrumentation. Such an equipment aims at simultaneously recording the polarized visibilities as well as reducing the instrumental bias to achieve the required accuracies (See Sect. 5.2). For the latter reason, polarimetric focal equipments separating the polarized interferograms must be advocated even for classical interferometers such as the VLTI or the Optical Very Large Array (OVLA, Labeyrie et al. 1987). Thus the "classical" High Angular Resolution observations (i.e. without 
Table 5. Limiting visual magnitudes for various accuracies on the visibility $\mathrm{d} V$ and various configurations of mono- and multispeckle mode

\begin{tabular}{ccccrl}
\hline $\mathrm{d} V$ & SNR & $N_{\mathrm{s}}$ & Coherence area & $M_{V}$ & Comments \\
& & & & & \\
\hline $1.0 \%$ & 150 & 1 & $\sigma=510^{-3} \mathrm{~m}^{2}$ & 4.0 & Monospeckle mode \\
$0.1 \%$ & 1500 & 1 & $\sigma=510^{-3} \mathrm{~m}^{2}$ & -1.0 & Monospeckle mode \\
$1.0 \%$ & 150 & 200 & $\sigma=510^{-3} \mathrm{~m}^{2}$ & 9.8 & Multispeckle mode (case of GI2T/REGAIN) \\
$0.1 \%$ & 1500 & 200 & $\sigma=510^{-3} \mathrm{~m}^{2}$ & 4.8 & Multispeckle mode (case of GI2T/REGAIN) \\
$1.0 \%$ & 150 & 1 & $s \pi R^{2}=018 \mathrm{~m}^{2}$ & 7.9 & Adaptive optics mode (case of GI2T/REGAIN) \\
$0.1 \%$ & 1500 & 1 & $s \pi R^{2}=018 \mathrm{~m}^{2}$ & 2.9 & Adaptive optics mode (case of GI2T/REGAIN) \\
$1.0 \%$ & 150 & 1 & $s \pi R^{2}=051 \mathrm{~m}^{2}$ & 9.0 & Adaptive optics mode (case of VLTI) \\
$0.1 \%$ & 1500 & 1 & $s \pi R^{2}=051 \mathrm{~m}^{2}$ & 4.0 & Adaptive optics mode (case of VLTI) \\
\hline
\end{tabular}

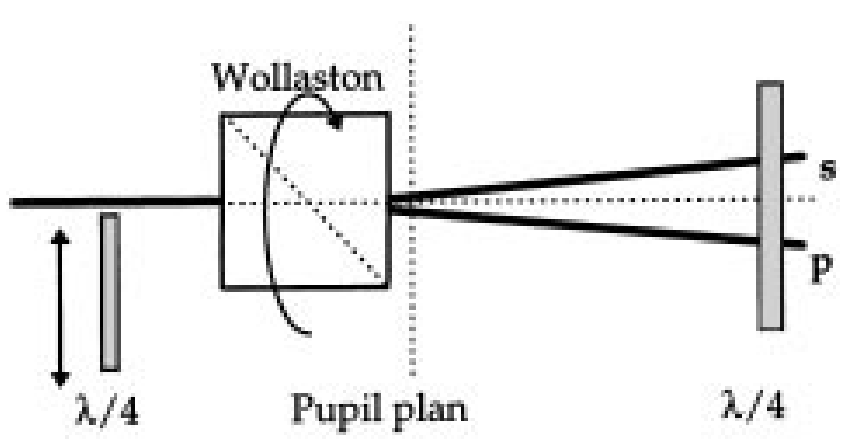

Fig. 8. Optical design of the polarimetric focal instrumentation of the GI2T/REGAIN Interferometer. The Wollaston prism can set two fixed positions $\left(0^{\circ}\right.$ and $\left.180^{\circ}\right)$. The wave-quarter plates are oriented at $45^{\circ}$ with respect to the Wollaston prism axes. The first one can be removed and enables to measure the circularly polarized components. The second one is fixed and lets optimize the global transmission of the interferometer (the grating of the following spectrograph greatly polarizes the linearly polarized components). The polarimeter can be removed for the measurements in natural light

polarimeter) would not be biased by instrumental polarization which dramatically degrades the fringe visibility at the combined focus (Rousselet- Perraut et al. 1996a). The principle of the GI2T/REGAIN polarimeter is given in Fig. 8 (Rousselet-Perraut et al. 1996b).

\subsection{Observational requirements}

Our study enables us to define the main steps of an interfero-polarimetric observing run:

i) Observations at large baselines (several tenth meters). For these spatial frequencies, the envelope contribution is very small and the visibility curve versus spatial frequency provides the envelope flux $F_{\text {env }}$ (See Sects. 3.2 and Stee et al. (1995) for the example of the Be star $\gamma$ Cassiopeiae). ii) Observations at short baselines $(10-20$ meters in the visible). For these spatial frequencies, the curve of the degree of polarized visibility versus spatial frequency allows to determine the exponent $\mathrm{k}$ of the electron distribution (See Sects. 3.3 and 4.3).

iii) Observations for various hour angles H. Given a baseline, observing at hour angles within $2-3$ hours around the meridian transit enables Earth-rotation synthesis: when $\mathrm{H}$ varies, the orientation of the baseline projected on the sky varies and the spatial frequency too. Thus we obtain a $2-\mathrm{D}$ information about the envelope geometry (angular dimensions and flattening $\varepsilon$ if any). Such High Angular Resolution observations lead to accurate flattening determinations (an accuracy of \pm 0.05 has been obtained by Quirrenbach et al. (1993) with the MARK III Interferometer).

For each step, "observations" obviously means that we record the three visibilities ( $V_{\text {nat }}$ without the polarimeter, $V_{/ /}$and $V_{\perp}$ with the polarimeter) for the studied object then for a standard polarimetric calibrator. Note that an unresolved source can also be used as a calibrator provided that its polarization degree remains less than $1 \%$ (Rousselet-Perraut 1997c).

\section{Conclusion}

This paper aimed at designing instrumental and observational constrains of interfero- polarimetry for studying matter distribution inside scattering environments. Adding a polarimetric mode to an interferometer extends its capacities to explore extended atmospheres in which intrinsic polarization becomes a non negligible observable. This implies to observe at small spatial frequencies (i.e. $\left.B / \lambda \approx 1 /\left(10 R^{*}\right)\right)$ and with a specific focal instrumentation separating the polarized interferograms and enabling an accurate calibration of the instrumental polarization. For spherical or ellipsoidal hot envelopes, an accuracy of $1 \%$ on the visibility is enough to put a lower limit to the 
exponent of the matter distribution, which is obviously compatible with existing instruments. Moreover this leads to limiting magnitudes $M_{v} \approx 4$ in a monospeckle mode and $M_{v} \approx 8-10$ in multispeckle modes, which permits to foresee the observation of many hot stars by this technique.

Our accuracy on the angular diameter is not enough to detect its variations with polarization. Thus the envelope dimensions can be determined by High Angular Resolution observations in natural light. Using Earthrotation synthesis allows to constrain the envelope flattening.

We have limited our study to pure single Thomson scattering and to an equatorial observation, which constitutes the most favorable case. Now, we should obviously integrate in our general formalism more realistic source functions (including opacities) and take into account multiple scattering or polarization inside emission lines (Be stars, ...) to provide a more detailed analysis of physical conditions in the envelope. For this purpose and to strongly constrain the geometry and the density of the envelope, interfero-polarimetry has to be used in conjunction with others techniques such as spectroscopic ones especially for Be stars (Waters 1986).

Of course interfero-polarimetry can be applied to future imaging arrays (Aime \& Roddier 1976; RousseletPerraut et al. 1997b) for which a polarimetric instrumentation must be advocated since it greatly reduces the instrumental bias. Moreover providing quasi instantaneous polarized images of the intensity maps, these future arrays (NPOI - Armstrong 1994, OVLA) would enable to detect local structures at the stellar surfaces or throughout circumstellar environments. As such, they would contribute to a better understanding of the mass-loss mechanisms and the stellar evolution theories.

Finally we can obviously foresee to extend this technique to other objects such as contact or semi-contact binaries where mutual reflection effect is at play, evolved red supergiants for studying grains and dusts scattering or absorption in molecular bands, magnetic stars for obtaining magnetograms and constraining the models of oblique rotators, ... But for these objects, limiting magnitudes can be constraining and besides adapted astrophysical models have to be developed to compute the flux contributions.

Acknowledgements. The author wants to greatly thank F. Perraut, F. Vakili, D. Mourard, Ph. Stee and F. Ménard for the discussions and their fruitful comments. The author is grateful to $\mathrm{K}$. Wood who refereed this paper and whose suggestions were very helpful. This work has been supported by the Collège de France.

\section{References}

Aime C., Roddier F., 1976, Opt. Commun. 19, 57-60

Armstrong J.T., 1994, "Progress on the Big Optical Array (BOA)", SPIE 2200, p. 62-70

Brown J.C., McLean I.S., 1977, A\&A 57, 141-149

Cassinelli J.P., Hoffman N.M., 1975, MNRAS 173, 789

Collett E., 1993, Polarized light - Fundamentals and applications. Marcel Dekker, New York

Françon M., 1966, Optical Interferometry. Academic Press, London

Coyne G.V., 1976, in Be and shell stars, Slettebak A., (ed.). IAU Symp. 70, Reidel, Dordrecht

Hanbury Brown R., Davis J., Allen L.R., 1974, MNRAS 168, 93

Labeyrie A., et al., 1987, "Multiple Aperture Interferometry: Towards the Optical Very Large Array", in Interferometric Imaging in Astronomy, NOAO/ESO Conf

Mazurkewich D., et al., 1991, AJ 101 (6), 2207-2219

Mourard D., et al., 1994, "REGAIN: A new optical beamcombiner for the GI2T", SPIE 2200, 593-598

Mourard D., et al., 1997, A\&A 317, 789-792

Paresce F., et al., 1996, The Messenger 83, 14-21

Percheron I., 1991, PhD Thesis, University of Nice

Perrin G., 1996, PhD Thesis, University of Paris VII

Poeckert R., Marlborough J.M., 1978, ApJ 220, 940

Quirrenbach A., Hummel C.A., Buscher D.F., et al., 1993, ApJ 416, L25

Roddier F., Léna P., 1984, J. Opt. (Paris) 15(4), 171-182

Roddier C., Roddier F., 1976, JOSA 66, 12, 1347-1350

Rousselet-Perraut K., et al., 1996a, Opt. Eng. 35, 10, 2943-2955

Rousselet-Perraut K., et al., 1996b, PhD Thesis, University of Marseille

Rousselet-Perraut K., et al., 1997a, A\&AS 123, 173-177

Rousselet-Perraut K., et al., 1997b, Opt. Eng. 36, 4, 980-987

Rousselet-Perraut K., et al., 1997c, "Calibration of the GI2T/REGAIN visibilities - Polarization effects" (private communication)

Rousset G., et al., 1992, "Adaptive optics partial correction simulation for two-telescope interferometry", in Beckers J.M., Merkle F. (eds.). High Resolution Imaging by Interferometry II, ESO Conf., Garching, October 1991, p. 1095

Serkowski, 1968, ApJ 154, 115

Serkowski, 1970, ApJ 160, 1083

Stee Ph., et al., 1995, A\&A 300, 219-238

Vakili F., 1981, A\&A 101, 352

Waters L.B.F.M., 1986, A\&A 162, 121-139

Wood K., et al., 1993, A\&A 271, 492-500

Wood K., et al., 1996, ApJ 461, 828-846 